\title{
Visual sources - List of illustrations
}

Cover: Egbert van Panderen (1581-1637?), Physician (from: "The medical practitioner as Christ, angel, man and devil”), Wellcome Collection, London.

Fig. 1 Joris Hoefnagel, Innsbruck with the castle of Ambras (after Alexander Colin), in: Civitates Orbis Terrarum, part 5, Cologne 1598, $\mathrm{n}^{\circ} 58$.

Fig. 2 Page from one of Georg Handsch's notebooks (Österreichische Nationalbibliothek, Vienna, Cod. 11183, fol. 434r).

Fig. 3 Michiel Jansz van Mierevelt, Anatomy lesson of Dr Willem van der Meer, 1617, Museum Prinsenhof, Delft.

Fig. 4 Bernard van Orley, Portrait of the physician Joris van Zeile, Musées royaux des Beaux-Arts de Belgique, Brussels.

Fig. 5 Statue of St Cosmas, with urine glass, Wellcome Collection, London.

Fig. 6 Rheubabarum in: Pietro Andrea Mattioli, I discorsi nelli sei libri di Pedacio Dioscoride Anazarbeo, Venice 1568, Wellcome Collection, London.

Fig. 7 Painful surgical treatment, oil painting by Gerrit Lundens, 1649, Wellcome Collection, London.

Fig. 8 Lukas van Leiden, Dentist, 1523, Wellcome Collection, London.

Fig. 9 Unknown painter, after Frans van Mieris (orig. 1657), The doctor's visit, Wellcome Collection, London.

Fig. 10 Distilling oven (balneum Mariae) in: Pietro Andrea Mattioli, Kreutterbuch, 1611, Universitätsbibliothek Erfurt, Sign. $13-\mathrm{MA} 2^{\circ} 23 \mathrm{t}$.

Fig. 11 Unknown artist, Guy de Chauliac performing a dissection, gouache after a $15^{\text {th }}$-century illuminated manuscript, Wellcome Collection, London.

Fig. 12 Francesco Segala, relief portrait of Archduke Ferdinand II, around 1580, Kunsthistorisches Museum, Vienna, KK 3085.

Fig. 13 Egbert van Panderen (1581-1637?), The medical practitioner as Christ, angel, man and 14 and devil, Wellcome Collection, London.

Fig. 15 Frans van Mieris, The doctor's visit, 1667, Paul Getty Museum, Los Angeles.

Fig. 16 Bath chamber with clients in a tub, on a sweating bench and undergoing cupping, Herzog August-Bibliothek, Wolfenbüttel, Cod. Guelf. 8.7. Aug. $8^{\circ}$, fol. 139r. 
